\section{Identification of male-specific active host plant volatiles for maize stem borer, Chilo partellus Swinhoe}

\author{
Niraj Guleria ${ }^{1}$, Suresh M. Nebapure ${ }^{1, *}$, \\ P. D. Kamala Jayanthi ${ }^{2}$, S. B. Suby ${ }^{3}$ and \\ P. Saravan Kumar ${ }^{2}$
}

${ }^{1}$ Division of Entomology, ICAR-Indian Agricultural Research Institute, New Delhi 110 012, India

${ }^{2}$ Department of Entomology and Nematology, ICAR-Indian Institute of Horticultural Research, Hessarghatta, Bengaluru 560 089, India ${ }^{3}$ ICAR-Indian Institute of Maize Research, Punjab Agricultural University Campus, Ludhiana 141004 , India

The present study was conducted to identify male-specific active plant volatiles from maize against Chilo partellus. The gas chromatography-electroantennogram detector evaluation of volatile extracts collected from maize plant through dynamic headspace collection revealed the presence of six electrophysiologically active volatiles for male, viz. toluene, 2-ethyl-1-hexanol, 4hydroxy-4-methyl-2-pentanone, 1,4-dichlorobenzene, $p$ isopropyl benzaldehyde (cuminaldehyde) and $p$-xylene. Electroantennogram analysis of selective synthetic volatiles against male moths revealed significantly higher response to geranyl acetate, cuminaldehyde, linalool and 2-ethyl-1-hexanol. Electroantennogram studies showed dose-dependent responses of male moths against geranyl acetate, cuminaldehyde, linalool and 2-ethyl-1-hexanol up to $50 \mu \mathrm{g}$, above which saturation of antennal response was noticed. These findings will help to study the synergistic activity of identified volatiles with pheromone compounds for developing more effective lure for $C$. partellus monitoring and mass trapping.

Keywords: Dynamic headspace collection, gas chromatography-electroantennogram detector, maize stem borer, male-specific active volatiles.

MAIZE stem borer Chilo partellus Swinhoe (Lepidoptera: Crambidae) is the most common oligophagous insect pest which feeds on grasses and cultivated cereals, especially maize and sorghum. In India, it has been reported to cause up to $80.4 \%$ damage in maize ${ }^{1}$. The current management strategies of $C$. partellus depend upon synthetic insecticides which are generally ineffective due to its cryptic feeding behaviour. Semiochemicals, which include pheromones and allelochemicals, are considered as a potential tool in pest management programmes. Though the sex pheromone components of female $C$. partellus, namely (Z)-11-hexadecenal and (Z)-11-hexadecenol have been identified ${ }^{2}$, they are not being widely used for monitoring or mass trapping of this pest, mainly due to the low response or attraction of male moths to the sex phero-

\footnotetext{
*For correspondence. (e-mail: smnebapure@iari.res.in)
}

mone lures. Generally, host plant volatiles play a significant role in host selection for feeding and oviposition by insect pests ${ }^{3}$. These volatiles have been proved to have synergistic effect when combined with pheromone compounds and can be utilized for improving the attraction efficiency of sex lures ${ }^{4}$. Therefore, the present study was aimed to identify male $C$. partellus-specific bioactive host plant volatiles from maize through detailed electrophysiological evaluations. The larvae of $C$. partellus were collected from maize fields located at Hapur, Uttar Pradesh, India $\left(28.7306^{\circ} \mathrm{N}, 77.7759^{\circ} \mathrm{E}\right)$, and reared on natural diet (baby-corn cobs) and subsequently on an artificial diet in controlled room condition (temperature $26^{\circ} \pm 2{ }^{\circ} \mathrm{C}$ and relative humidity $\left.75 \pm 5 \%\right)^{5}$. To obtain unmated males and females, pupa once formed were isolated through sexing and kept in separate glass jars (15 cm diameter and $20 \mathrm{~cm}$ height) till adult emergence. For evaluation, authentic chemical standards of synthetic volatile compounds were used in this study ( $\geq 98 \%$ pure: hexanal, (Z)-3-hexen-1-ol, benzyl acetate, methyl salicylate, geranyl acetate, $(Z)-3$-hexen-1-yl acetate, benzaldehyde, phenyl acetaldehyde, acetophenone, methyl benzoate, eugenol, 2-ethyl-1-hexanol, 4-hydroxy-4-methyl-2-pentanone, methyl anthralinate, cuminaldehyde (4-isopropylbenzaldehyde); 97\% pure: linalool, limonene, $p$-xylene; $\geq 90 \%$ pure: $\alpha$-terpinolene; $\geq 80 \%$ pure: $\beta$-caryophyllene) were procured from Sigma-Aldrich, India.

Maize, Zea mays L. (variety HQPM-1) was grown in a glasshouse and covered with a net. Healthy plants (1520 days-old) were used for volatile collection through the dynamic headspace collection method $^{6}$. The volatiles were collected for $6 \mathrm{~h}$ and the entrapped volatiles were eluted with $300 \mu \mathrm{l}$ of HPLC-grade dichloromethane (DCM; CDH, India) and stored at $-20^{\circ} \mathrm{C}$. Volatile collections were repeated six times, each with a fresh set of the plant. Gas chromatography-electroantennogram detector (GC-EAD) and gas chromatography-mass spectrometry (GC-MS) analysis of the extracts was done to identify the compounds perceived by $C$. partellus adults (both male and female $)^{7}$. Peaks eluting from the GC column were considered to be active if they elicited EAG activity in three or more of the five-coupled runs carried out. The traces from flame ionization detector (FID) and EAD were plotted and the matching peaks along with their retention times were measured. The data was analysed using a customized software package (Syntech, Germany).

Further, EAG responses from only male $C$. partellus were recorded against GC-EAD active volatiles along with volatile cues of other host plants, namely sorghum (Sorghum bicolor), Napier grass (Pennisetum purpureum) and blue thatching grass (Hyparrhenia tamba), which are reported to be attractive to C. partellus ${ }^{8-10}$. The EAG setup and odour delivery procedure in this study were similar to those of Sen et al. ${ }^{11}$, with some modifications. The antenna was excised and fixed to a gel electrode using Spectra 360 gel. In the first step, all the volatiles were 


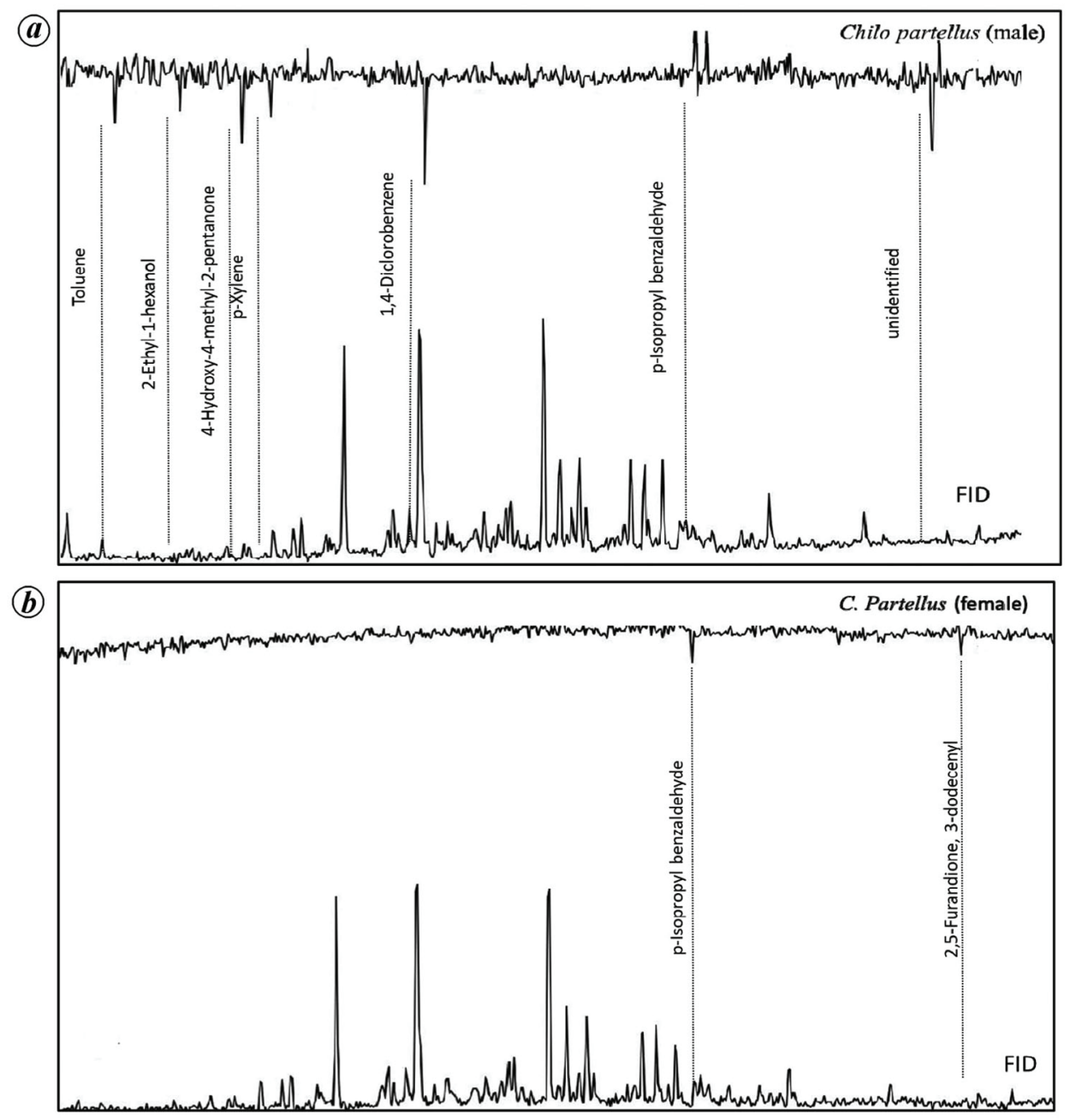

Figure 1. GC-EAD responses of (a) unmated male and (b) female Chilo partellus to constituents of the maize headspace extracts. The upper traces in each figure represent signals from EAD and the lower traces represent signals from the FID.

screened for EAG responses at a single dose $(50 \mu \mathrm{g})$. The synthetic compounds were diluted using hexane. Each dose was replicated five times and each time a different antenna from a different moth was used. Hexanal served as a reference standard for testing the consistency of antennal responses after every three compounds. Similarly, a solvent was presented after every three compounds. The EAG responses for control were averaged, and subtracted from those obtained against the compounds. The EAG responses were expressed relative to hexanal using the following formula

$$
r \mathrm{EAG}(\%)=\frac{x \mathrm{EAG}}{s \mathrm{EAG}} \times 100,
$$

where $r \mathrm{EAG}$ is the relative EAG response of compound $x, x$ EAG the amplitude $(\mathrm{mV})$ of the EAG response to compound $x$ and sEAG is the EAG amplitude of the reference compound (hexanal). EAG 2000 software (version
$2.7 \mathrm{c}$, Syntech, Germany) was used for analysis. The volatiles which elicited significant response from male antennae were evaluated individually for their dose-response assay at five doses, viz. 1, 5, 10, 50 and $100 \mu \mathrm{g}$. The mean relative EAG responses were subjected to one-way ANOVA (SPSS 16.0) and the means were separated using Tukey's test.

GC-EAD analysis revealed that a total of six compounds in the maize plant volatiles, namely toluene, 2-ethyl-1-hexanol, 4-hydroxy-4-methyl-2-pentanone, 1,4dichlorobenzene, $p$-isopropyl benzaldehyde and $p$-xylene consistently elicited EAD responses from the antennae of unmated male (Figure $1 a$ ) and unmated female gave only response to $p$-isopropyl benzaldehyde and 2,5-furandione, 3-dodecenyl (Figure $1 b$ ). This shows that headspace volatiles which give response to unmated male are specific, except $p$-isopropyl benzaldehyde for which unmated female also responded. Further, EAG studies revealed significantly higher mean relative EAG responses of 


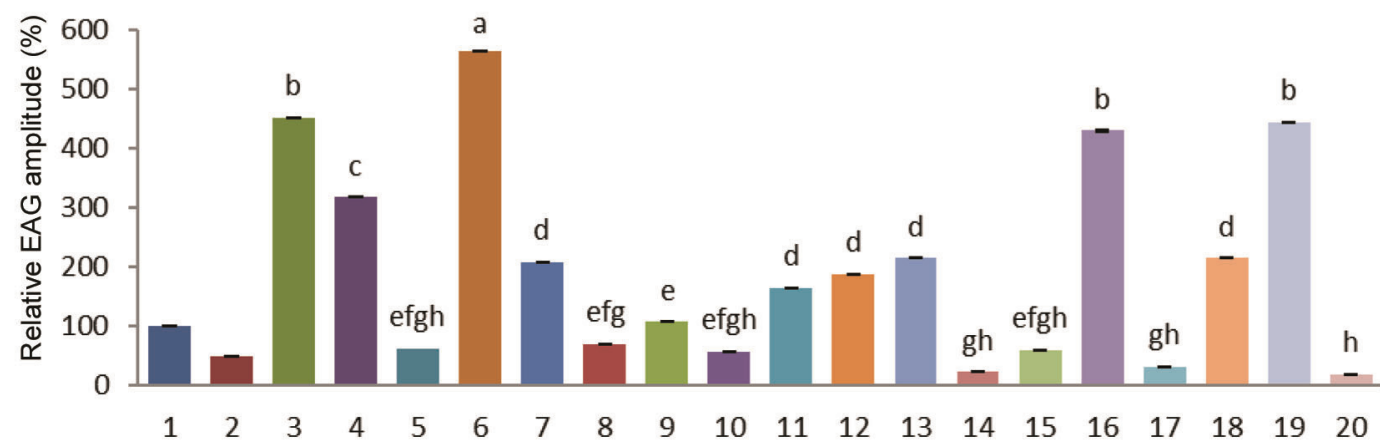

Figure 2. Relative EAG responses of $C$. partellus male to maize plant volatiles. Bars with different letters show significantly different mean mortality (\%) at $P<0.05$ by Tukey's test. 1, Hexanal; 2, (Z)-3-hexen-1-ol; 3, Linalool; 4, Benzyl acetate; 5, Methyl salicylate; 6, Geranyl acetate; 7, $\beta$-caryophyllene; 8, (Z)-3-hexen-1-yl acetate; 9, Benzaldehyde; 10, Phenyl acetaldehyde; 11, Acetophenone; 12, Methyl benzoate; 13, Eugenol; 14, Limonene; 15, $\alpha$-terpinolene; 16, 2-Ethyl-1-hexanol; 17, 4-Hydroxy-4-methyl-2-pentanone; 18, Methyl anthralinate; 19, $P$ isopropyl benzaldehyde (Cuminaldehyde); 20, $p$-xylene.
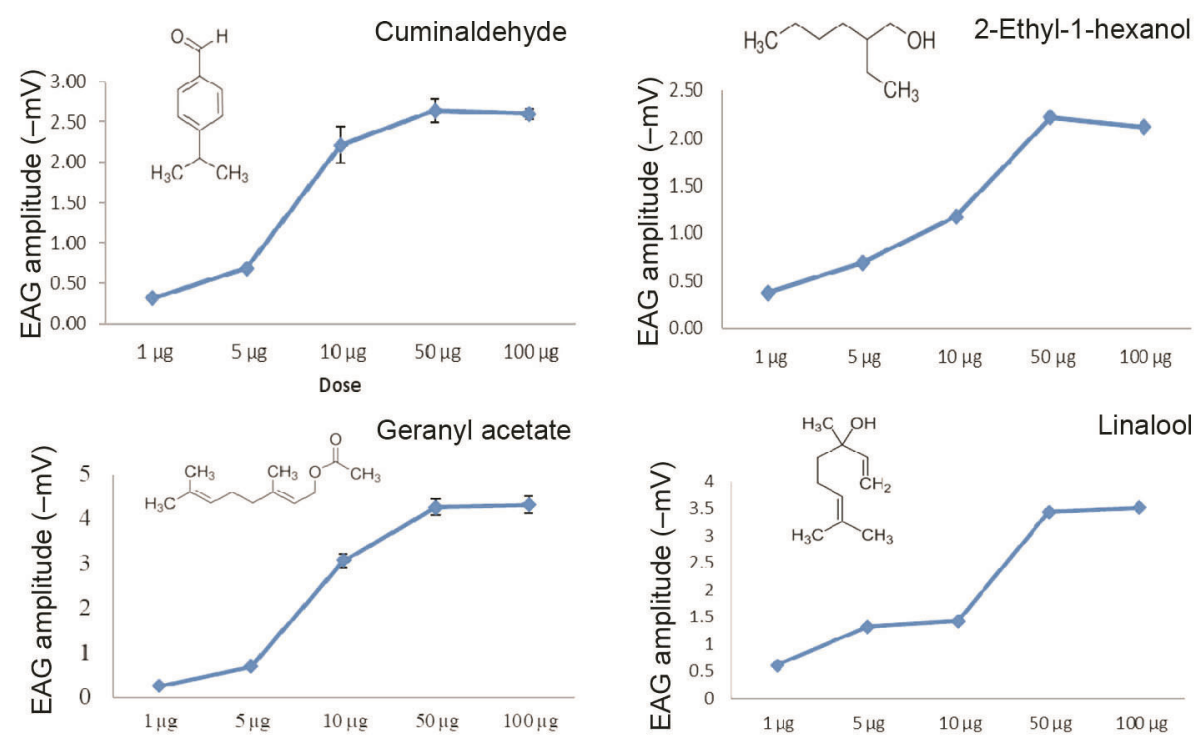

Figure 3. Dose-dependent EAG responses of $C$. partellus male to selected plant volatiles.

unmated male $C$. partellus to geranyl acetate $(562.95 \%)$, followed by linalool $(450.68 \%)$, cuminaldehyde $(444.23 \%)$ and 2-ethyl-1-hexanol (429.53\%; $F=266.11$; $P<0.05$; Figure 2). The response to geranyl acetate was fivefold more than the reference compound, viz. hexanal. These significantly different response-eliciting compounds, viz. geranyl acetate, linalool, cuminaldehyde and 2-ethyl-1-hexanol were studied further for their dosedependent EAG responses. It was observed that as the dose increased from 1 to $50 \mu \mathrm{g}$, the EAG amplitude also increased, but after $50 \mu \mathrm{g}$, there was no increase in EAG amplitude. The response elicited for all the compounds at each dose was found to be significantly different (geranyl acetate, $F=116.19 ; \quad P<0.05, \quad$ linalool, $F=1246.1$, $P<0.05$; cuminaldehyde, $F=48.471, P<0.05$ and 2 ethyl-1-hexanol, $F=3762.3, P<0.05$; Figure 3 ).
Significance of host plant volatiles for insect pests has been studied by several workers. It is now well known that insects use plant volatiles as one of the long-range olfactory cues to locate the host plant $^{12}$ and sometimes to locate mates ${ }^{13}$. Male-specific plant volatiles have major significance for developing synergistic lures in combination with sex pheromones, as it is suggested that plant volatiles enhance the attraction of pheromone compounds thereby helping in developing more effective trapping techniques $^{14,15}$. The main objective of this study was to identify male-specific maize plant volatiles which further can be explored for pheromone and plant volatile synergism studies against $C$. partellus. In the present study, toluene was reported as one of the electrophysiologically active volatiles for male moths of $C$. partellus, and has also been previously demonstrated from dynamic 
headspace collections of S. bicolor, Z. mays, P. purpureum and $H$. tamba. Similarly, another EAD-active compound, 2-ethyl hexanol, has been reported as an electrophysiologically active component for male fruit piercing moth, Eudocima materna from fruit volatiles ${ }^{16}$. The attractiveness of bioactive compounds that elicited antennal response in the present study through GCEAD/EAG analyses needs to be further ascertained using detailed olfactometer bioassays. Although geranyl acetate was found to elicit maximum response from $C$. partellus males, it has been reported to reduce in male response by $34.6 \%$ Spodoptera littoralis. Linalool has been found to synergize the pheromone of Spodoptera exigua (Hubner), Helicoverpa zea (Boddie) and Cydia pomonella (Linnaeus), but not for Spodoptera litura (Fab.) ${ }^{17}$ and Spodoptera littoralis (Boisd.) ${ }^{18}$. Hence, before specifying the functional role of these volatiles in behaviour manipulation of $C$. partellus males and to using them for developing synergistic lures in combination with pheromone fractions, further laboratory studies involving olfactometer and wind-tunnel assays coupled with field evaluation are needed.

1. Sethuraman, V. and Narayanan, K., Biological activity of nucleopolyhedrovirus isolated from Chilo partellus (Swinhoe) (Lepidoptera: Pyralidae) in India. Asian J. Exp. Biol. Sci., 2010, 1, 325330

2. Nesbitt, B. F., Beevor, P. S., Hall, D. R., Lester, R., Davies, J. C. and Reddy, K. S., Components of the sex pheromone of the female spotted stalk borer, Chilo partellus (Swinhoe) (Lepidoptera: Pyralidae): identification and preliminary field trials. J. Chem. Ecol., 1979, 5(1), 153-163.

3. Sole, J., Sans, A., Riba, M. and Guerrero, A., Behavioural and electrophysiological responses of the European corn borer Ostrinia nubilalis to host-plant volatiles and related chemicals. Physiol. Entomol., 2010, 35(4), 354-363.

4. Reddy, G. V. P. and Guerrero, A., Interactions of insect pheromones and plant semiochemicals. Trends Plant Sci., 2004, 9, $253-$ 261.

5. Siddiqui, K. H., Sarup, P., Panwar, V. P. S. and Marwaha, K. K., Evolution of base ingredients to formulate artificial diets for the mass rearing of Chilo partellus (Swinhoe). J. Entomol. Res., 1977, 1, 117-131.

6. Tholl, D., Boland, W., Hansel, A., Loreto, F., Rose, U. S. and Schnitzler, J. P., Practical approaches to plant volatile analysis. Plant J., 2006, 45(4), 540-560.

7. Pagadala Damodaram, K. J., Gadad, H. S., Parepally, S. K., Vaddi, S., Ramanna Hunashikatti, L. and Bhat, R. M., Low moisture stress influences plant volatile emissions affecting herbivore interactions in tomato, Solanum lycopersicum. Ecol. Entomol., 2021, 46(3), 637-650.

8. Chamberlain, K., Khan, Z. R., Pickett, J. A., Toshova, T. and Wadhams, L. J., Diel periodicity in the production of green leaf volatiles by wild and cultivated host plants of stemborer moths, Chilo partellus and Busseola fusca. J. Chem. Ecol., 2006, 32(3), 565-577.

9. Birkett, M. A., Chamberlain, K., Khan, Z. R., Pickett, J. A., Toshova, T., Wadhams, L. J. and Woodcock, C. M., Electrophysiological responses of the lepidopterous stemborers Chilo partellus and Busseola fusca to volatiles from wild and cultivated host plants. J. Chem. Ecol., 2006, 32(11), 2475-2487.
10. Magara, H. J., Mutyambai, D. M., Charles, M. A., Otieno, S. A., Nyaga, T. M., Niassy, S. and Khan, Z. R., Responses of stem borer Chilo partellus to volatiles emitted by maize landraces exposed to signal grass (Brachiaria brizantha). J. Plant Interact., 2020, 15(1), 345-357.

11. Sen, A., Raina, R., Joseph, M. and Tungikar, V. B., Response of Trichogramma chilonis to infochemicals: an SEM and electrophysiological investigation. BioControl, 2005, 50(3), 429-447.

12. Bruce, T. J., Wadhams, L. J. and Woodcock, C. M., Insect host location: a volatile situation. Trends Plant Sci., 2005, 10(6), 269274.

13. Von Arx, M., Schmidt-Busser, D. and Guerin, P. M., Plant volatiles enhance behavioral responses of grapevine moth males, Lobesia botrana to sex pheromone. J. Chem. Ecol., 2011, 38(2), 222-225.

14. Landolt, P. J. and Phillips, T. W., Host plant influences on sex pheromone behavior of phytophagous insects. Аnпu. Rev. Entomol., 1997, 42, 371-391.

15. Knight, A. L., Hilton, R. and Light, D. M., Monitoring codling moth (Lepidoptera: Tortricidae) in apple with blends of ethyl (E, Z)-2,4-decadienoate and codlemone. Environ. Entomol., 2005, 34(3), 598-603.

16. Mallikarjun, K. R. M., Thippaiah, M., Raghavendra, A., Sharma, J. and Chakravarthy, A. K., Role of fruit volatiles and sex pheromone components in mate recognition in fruit piercing moth Eudocima materna Linnaeus (Lepidoptera: Erebidae). J. Entomol. Zool. Stud., 2019, 7(3), 1381-1387.

17. Fang, Y., Zeng, R., Lu, S., Dai, L. and Wan, X., The synergistic attractiveness effect of plant volatiles to sex pheromones in a moth. J. Asia Pac. Entomol., 2018, 21(1), 380-387.

18. Renou, M., Party, V., Rouyar, A. and Anton, S., Olfactory signal coding in an odor background. Biosystems, 2015, 136, 35-45.

Received 28 March 2021; revised accepted 30 June 2021

doi: $10.18520 / \mathrm{cs} / \mathrm{v} 121 / \mathrm{i} 4 / 578-581$

\section{Mites: an emerging problem for bumblebees in the Indian Himalayan Region}

\section{Rifat Husain Raina ${ }^{1, *}$, Babu Saddam ${ }^{1}$, Aejaz H. Parrey ${ }^{1,3}$, Purnima Pathak ${ }^{1}$ and Kailash Chandra ${ }^{2}$}

${ }^{1}$ Desert Regional Centre, Zoological Survey of India, New Pali Road, Jodhpur 342 005, India

${ }^{2}$ Zoological Survey of India, M-block, New Alipore 700 053, India ${ }^{3}$ Department of Zoology, School of Biosciences and Biotechnology, Baba Ghulam Shah Badshah University, Rajouri 185 234, India

To increase crop yield in the high altitude ecosystem in the Indian Himalayan Region (IHR), bumblebees are highly valued insects. An unwanted mites association with bumblebees is an example of a serious threat for the conservation of high-altitude agro-forestry

*For correspondence. (e-mail: raina.rifat@zsi.gov.in) 